\title{
An Integrated Amperiometric Sensor for in situ Environmental Monitoring
}

\author{
Angan Das, Prashant Bhadri, Fred R. Beyette Jr. \\ Dept. of ECECS, College of Engineering \\ University of Cincinnati \\ Cincinnati, OH, USA \\ Email: dasan@ececs.uc.edu
}

\author{
Am Jang ${ }^{1}$, Paul Bishop ${ }^{1}$, William Timmons ${ }^{2}$ \\ ${ }^{1}$ Dept. of CEE, College of Engineering \\ University of Cincinnati \\ Cincinnati, OH, USA \\ ${ }^{2}$ Entera Tech Inc. \\ Columbus, $\mathrm{OH}$, USA
}

\begin{abstract}
In the modern era, some of the most serious causes for public health problems can be attributed to environmental pollution. Proper monitoring of environmental conditions helps us to control the pollution in an effective manner. This paper introduces a robust, self-contained, inexpensive integrated amperiometric microelectrode sensor that can be used for in situ environmental monitoring. The work primarily focuses on the design, implementation and integration of an amperiometric CMOS sensor chip with a microelectrode sensor device in order to measure the current flowing through the working probe (microelectrode sensor) and the reference probe, when they are immersed in the test solution. The magnitude of the current signal, of the order of nanoamperes, is shown to be dependent on the characteristics (oxygen content) of the solution being evaluated by the system. The integration of the microelectrode sensor with the sensor chip is done with the help of a Printed Circuit Board. The microelectrode sensor device may be replaced by a NEMS based sensor device for applications requiring further miniaturization, like that in the biomedical field where the solution under test may be a biofilm .
\end{abstract}

Keywords - amperiometric, sensor, microelecrode, noise

\section{INTRODUCTION}

In recent times, environmental pollution has become one of the most detrimental factors contributing to public health problems. To keep the pollution levels under control, one needs to properly monitor the environmental conditions at a specific location. Substantial monitoring ensures constant removal of the toxicants or pollutants. This requires measurement of the concentration of the various pollutants both in aqueous and soil mediums. Mostly, the measurements are made on samples extracted from the related site. This is often not acceptable for sites that require constant monitoring or in situ monitoring, i.e. monitoring of the conditions at the actual location rather than measurements with the bulk samples collected. The applications requiring accurate in situ monitoring encompass a large number of areas including the monitoring of waste water treatment reactors, water distribution systems and stream or lake sediments. In situ monitoring also finds applications in the manufacturing and chemical production industry, the healthcare industry for medical diagnosis, pharmaceutical manufacturing, biological research and the food industry for monitoring bioreactors and fermentation processes. This kind of monitoring necessitates microelectrode sensors that can sense the external environmental conditions in a proper and efficient manner. The microelectrode sensors that have been developed till now are fragile and difficult to use for in situ measurements. Further, they pose problems in manufacturing and are susceptible to electrical interference or noise. All these factors constrain their use to laboratory environments under highly controlled conditions. This work introduces a robust, self-contained, inexpensive, amperiometric microelectrode sensor system that has integrated signal processing circuitry so that it can be used in situ in various harsh environments. From an upper system level perspective, the environmental sensor consists of two major components. The first one is a field deployable microelectrode sensor device that had been designed and implemented. On application of a suitable potential across the sensor device, a current signal is produced that is passed on to the second component, the sensor chip. The chip amplifies and processes the signal, eliminating the coupled environmental noise as far as possible. It is shown that the output current signal produced by the sensor chip varies with a change in the external conditions in which the probes are placed. The chip and the measuring microelectrode probes are embedded in a Printed Circuit Board to produce a fully integrated system.

The rest of the paper is arranged into the following sections. Section II introduces the design of the amperiometric circuitry for sensing the current signal. It also discusses the PCB that has been designed and fabricated to integrate the sensor chip with the sensing probes. In section III, we detail the amperiometric testing procedure adopted. Section IV reports the results and Section V finally concludes the work.

\section{AMPERIOMETRIC CIRCUIT DESIGN}

The sensor chip contains the required circuitry for amperiometric measurement with the microelectrode probes. It

This work has been supported by the National Science Foundation (Grant \# BES-0228603) and the National Institute of Environmental Health Sciences (Grant \# 5R43ES011891-02). 


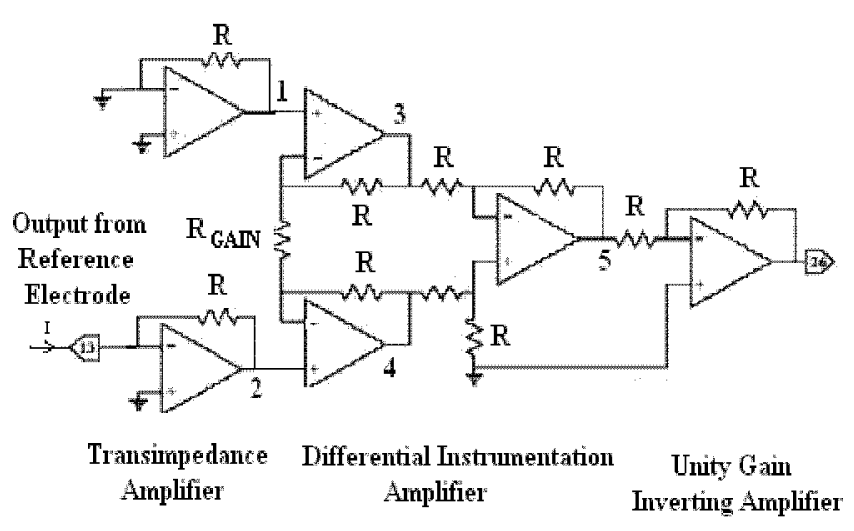

Figure 1. Amperiometric Circuit

is a 40 pin DIP chip has been designed and laid out using the Tanner Tools L-Edit layout editor in the $1.5 \mathrm{um}$ process and fabricated through the MOSIS foundry [3]. Fig. 1 shows the amperiometric circuit in the chip. It comprises of the following three stages connected in succession.

\section{A. Transimpedance Amplifer}

The transimpedance amplifier converts the current (I), sensed by the working electrode that is connected to Pin_13 of the sensor chip, into an equivalent voltage through the resistance $\mathrm{R}$. The other transimpedance amplifier has its input pin (Pin_14) grounded and thereby provides a $0 \mathrm{~V}$ reference voltage at node $1\left(\mathrm{~V}_{1}\right)$.

\section{B. Differential Instrumentation Amplifier}

The transimpedance amplifier is followed by a buffered differential amplifier. The variable resistance $\mathrm{R}_{\mathrm{GAIN}}$, provided from a potentiometer, is typically set to $1 \mathrm{k} \Omega$. The voltage drop between points 3 and $4, V_{3-4}$ is given by:

$$
\mathrm{V}_{3-4}=\left(\mathrm{V}_{2}-\mathrm{V}_{1}\right)\left(1+2 \mathrm{R} / \mathrm{R}_{\mathrm{GAIN}}\right)
$$

The regular differential amplifier following the buffered differential amplifier takes the voltage drop $\mathrm{V}_{3-4}$ and amplifies it further. However, owing to all the resistors used being of value $1 \mathrm{k}-\Omega$, the gain of the regular differential amplifier is equal to unity. The advantage of the differential amplifier lies in the fact that the overall gain can be varied by varying only a single resistor $\mathrm{R}_{\text {GAIN }}$. The other advantages relate to its high input impedance, high common mode rejection ratio (CMRR) and noise elimination capability. The output differential voltage is obtained at Pin_26.

\section{Unity Gain Inverting Amplifier}

The unity gain inverting amplifier is used in the last stage of the amperiometric circuit. It has both the resistors of value $1 \mathrm{k} \Omega$. This circuit functions as a buffer, helping in impedance matching and signal isolation.

\section{PRINTED CIRCUIT BOARD}

The chip is meant to sense current in the range of nanoamperes during amperiometric testing of the sensor system. Bread board testing of the chip introduces a lot of external noise, a major portion of which is due to the breadboard connection grid. In order to eliminate the noise effects due to wire connectivity as far as possible and to avoid external connection of the active elements like voltage supply as well as passive elements like resistors required for chip operation, a 2-layered Printed Circuit Board (PCB) has been designed using the Express PCB software [4] and fabricated through their foundry service. The main components of the PCB comprise of a power supply, voltage regulation circuitry and ribbon cable connectors for providing the input/output interface. It also consists of DIP sockets for holding the sensor electrodes, sensor chip and for providing necessary wire connectivity.

\section{TEST PROCEDURE}

\section{A. Amperiometric Testing}

The purpose of the amperiometric circuit is the measurement of current. But the circuit is so designed that it produces an output voltage. This owes to the reason that accurate measurement of the expected value of the current, being of the order of nanoamperes, poses a challenge even with a precise picoammeter, due to the noise levels introduced. On the contrary due to the high gain of the circuit, the voltage produced is of a higher magnitude and hence convenient to measure at the output pin (Pin_26). Now to know the current flowing from the voltage signal measured, we need to calibrate the circuit prior to any measurement. This is achieved by providing known values of current (0.5-100nA) at the input (Pin_14) and measuring the output voltage at Pin_26 with a digital multimeter, monitored using the LABVIEW measurement software program. The results demonstrate that the voltage obtained bears a second order fit to the current as given in (2) with the value of the degree of determination $\left(\mathrm{R}^{2}\right)$ equal to 0.9894 , which is statistically acceptable.

$$
I=\left(7.54327^{*} 10^{-7}\right) V^{2}-\left(1.39962^{*} 10^{-6}\right) V+\left(6.49811^{*} 10^{-7}\right)
$$

The block diagram of the test setup is given in Fig. 2. The variable bias potential needed to drive the current through the probes is provided from the $3 \mathrm{~V}$ battery supply and fed through the potentiometer on the PCB. This voltage is passed through the voltage follower circuit and is applied across the working and reference electrodes placed in chlorine dioxide $\left(\mathrm{ClO}_{2}\right)$ solution. $\mathrm{ClO}_{2}$ solution is chosen because of its stable oxygen content, which is actually responsible for the current flow in amperiometric testing. The concentration of the $\mathrm{ClO}_{2}$ solution is varied from $5 \mathrm{ppm}$ to $25 \mathrm{ppm}$ in steps of $5 \mathrm{ppm}$. For each of these concentrations, the bias potential is varied from $0.05 \mathrm{~V}$ to $0.15 \mathrm{~V}$ in steps of $0.025 \mathrm{~V}$.

\section{B. Noise Measurement}

One of the main objectives of the work is that the sensor chip and the PCB designed should eliminate external interference noise effects as far as possible. Therefore to ensure the proper elimination of noise, two kinds of testing are adopted. 


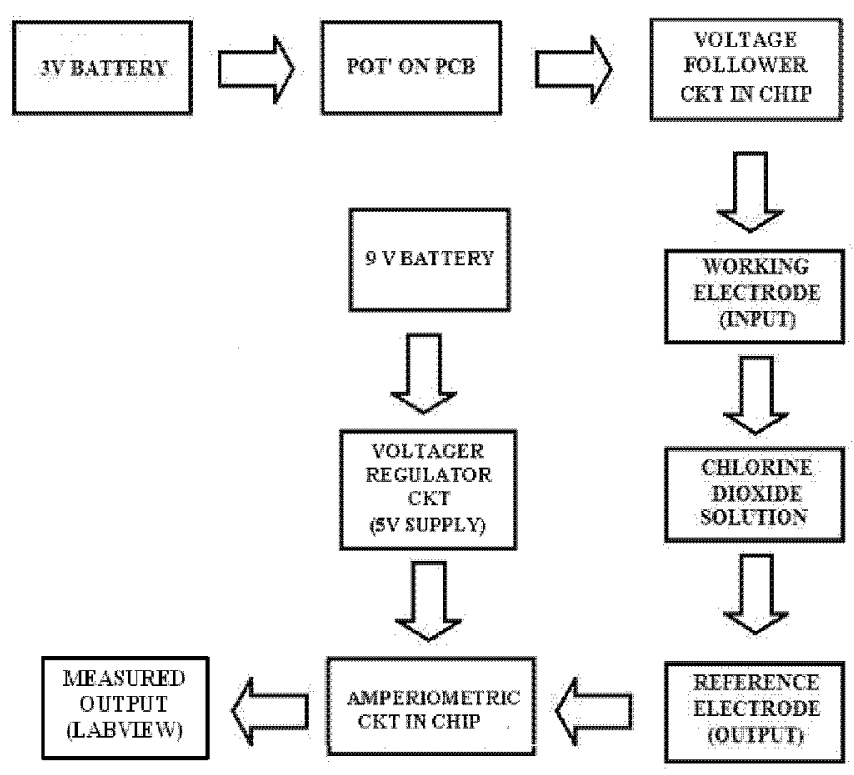

Figure 2. Schematic layout of amperiometric test setup

They are:

- All of the testing equipments and the probes are placed outside the Faraday's cage.

- All of the testing equipments and the probes are placed inside the Faraday's cage.

Moreover, to verify the appropriateness of the PCB in eliminating noise on top of that already eliminated by the chip, both of the above noise measurement procedures are adopted for two different kinds of situations. They are:

- Chip placed on a conventional breadboard.

- Chip placed on the PCB.

The rms values of noise for the different test situations are obtained with a precision multimeter and monitored using an automated LABVIEW measurement software program.

\section{RESULTS AND DISCUSSIONS}

\section{A. Amperiometric Measurement}

The results of amperiometric testing for both the situations, outside and inside Faraday's cage, are shown in Figs. 3(a) and 3(b) respectively. The results show that for a constant bias potential, with an increase in the concentration of the solution, the current flowing through the probes gradually increases. The reason for this kind of behavior is that, with an increase in solution concentration, the oxygen content of the solution increases which in turn decreases the resistance offered by the solution, thereby increasing the current that flows through it. The results also indicate that for a particular strength of the solution, the current flowing through it gradually increases as the bias potential increases. The plots show that the currents in

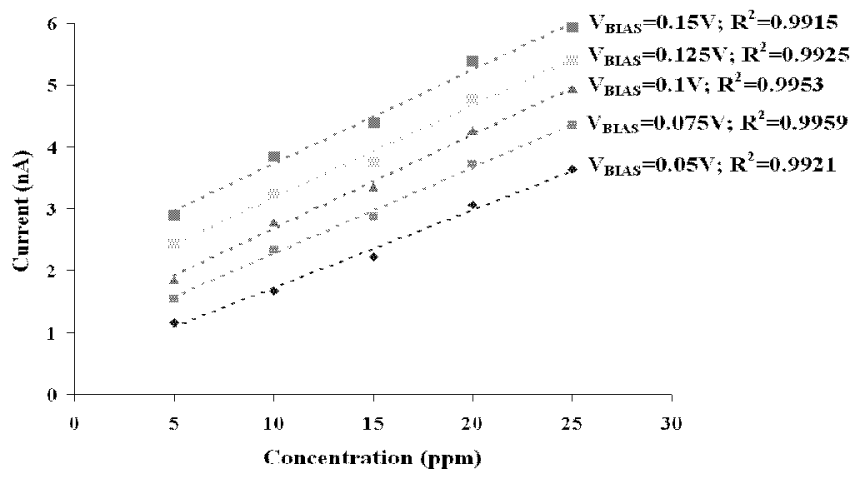

Figure 3(a). Plot of current vs. solution strength for different values of applied bias potential ( $0.05-0.15 \mathrm{~V})$ outside Faraday's cage

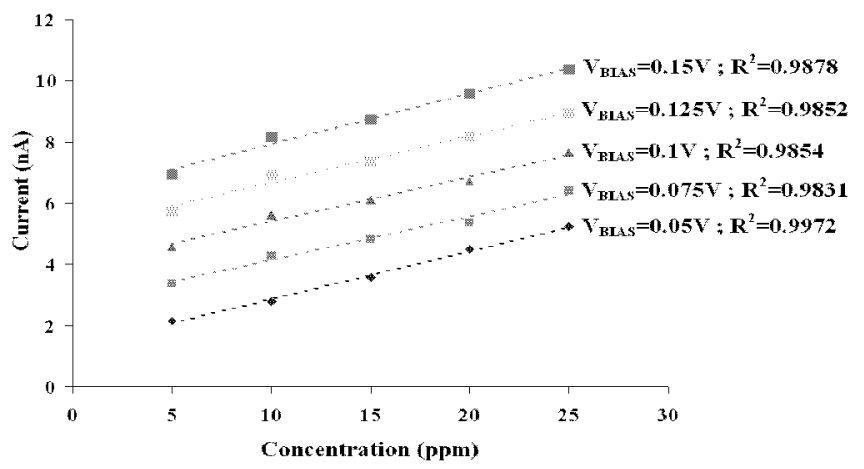

Figure 3(b). Plot of current vs. solution strength for different values of applied bias potential $(0.05-0.15 \mathrm{~V})$ inside Faraday's cage

both the situations bear straight line fits to increasing values of the solution concentration with statistically acceptable values of coefficients of determination $\left(\mathrm{R}^{2}\right)$. The surrounding temperature during amperiometric testing was 21 degrees Celsius. It is also to be noted that the results obtained without Faraday's cage are comparable to that obtained with the shielding provided. The sensor chip thereby eliminates the need of any external shielding.

\section{B. Transient Response}

The transient behavior for the probe measurements are taken for a time period ranging from 0-300 seconds to note the change in behavior of the probes over time. The response of the probes for a particular test condition is shown in Fig. 4 for convenience. It is seen that after a short transient period, the current starts flowing in the proper direction and attains a steady state value. In the example provided, the response time for the probe, i.e. the time required to attain the final steady state value is chosen to be that time after which the current remains within $\pm 25 \%$ of the mean value $(3.25 \mathrm{nA})$. The time is recorded to be 25 seconds in this case. In general, the currents obtained in the various test cases for amperiometric testing attained the steady state value within 30 seconds after the probes are placed in the solution. This shows that the response time of the fabricated probes is quite appreciable 


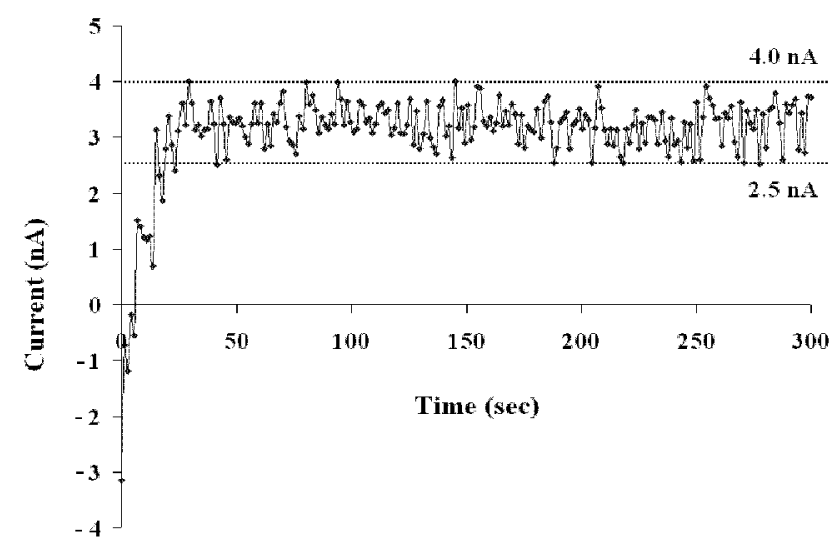

Figure 4. Transient response of the microelectrode sensor system in the $\mathrm{ClO}_{2}$ solution (Specific conditions: Applied bias potential $=0.125 \mathrm{~V}$, Conc. of solution $=10 \mathrm{ppm}) ; \mathrm{T}=300 \mathrm{~s}$

compared to that obtained with commercial versions where it is of the order of several minutes.

\section{Noise Measurement Results}

Exhaustive measurements are done to estimate noise throughout the whole test procedure. The noise measurement

TABLE I. AMPERIOMETRIC NOISE MEASUREMENTS

\begin{tabular}{|c|c|c|}
\hline \multirow{2}{*}{$\begin{array}{c}\text { Platform } \\
\text { (Setup) }\end{array}$} & \multicolumn{2}{|c|}{$\begin{array}{c}\text { Mean of the rms values of noise signal } \\
\text { (mV) }\end{array}$} \\
\cline { 2 - 3 } & $\begin{array}{c}\text { Outside Faraday's } \\
\text { cage }\end{array}$ & $\begin{array}{c}\text { Inside Faraday's } \\
\text { cage }\end{array}$ \\
\hline $\begin{array}{c}\text { With Sensor } \\
\text { Chip only }\end{array}$ & 15.82 & 10.03 \\
\hline $\begin{array}{c}\text { With Sensor } \\
\text { Chip + PCB }\end{array}$ & 8.38 & 3.78 \\
\hline
\end{tabular}

results are shown in Table $I$. The rms value of noise is measured continuously over the specified time period $(300 \mathrm{~s})$ using the LABVIEW measurement program and the average or mean is reported below. The external noise during the test had a mean rms value of $32.65 \mathrm{mV}$. It can be concluded from the results obtained that the chip helps in eliminating noise to a great extent. The PCB helps to reduce it further. Moreover, comparable results obtained inside and outside the Faraday's cage prove the robustness of the integrated microelectrode sensor in doing away with the necessity of any kind of external shielding mechanism. The integrated sensor system produces a noise-immune system that maintains the signal integrity.

\section{CONCLUSION}

The paper introduced a portable microelectrode based integrated amperiometric sensor system used for in situ environmental monitoring. The amperiometric circuit measures the current flowing through the sensor probes and helps in evaluating the environmental conditions around the probes. The sensor chip along with the PCB eliminates the effects of external noise to a great extent. The microelectrode sensor device may be replaced by a NEMS based device in applications such as biomedical devices where the solution under test may be a biofilm.

\section{REFERENCES}

[1] Am Jang, et al, "Fabrication of miniaturized redox potential probe for in situ environmental monitoring", Journal of Environ. Sci. and Technology, vol. 39, pp. 6191-6197, 2005.

[2] J.-H. Lee et al., "Microelectrode Arrays for In Situ Environmental Monitoring," in Proc. of Transducers '05, Seoul, Korea, June, 2005.

[3] http://www.mosis.org/

[4] http://www.expresspcb.com

[5] C. Gao et al., "A fully integrated biosensor array for measurement of metabolic parameters in human blood", Proc. of IEEE-EMBS Special Topic Conference on Microtechnologies in Medicine and Biology, Madison, pp. 223-226, May 2002

[6] M.Dutta, "Design of an integrated detection system for the characterisation of a biosensor array", M.S. thesis, University of Cincinnati, Cincinnati, OH, USA, 2003. 